\title{
Synthesis of gold nano-plates by mercaptide thermolysis in poly(vinyl acetate)
}

\author{
G. Cardone 1 , G. Carotenuto ${ }^{2}$, A. Longo 1, P. Perlo L L. Ambrosio $^{2}$ \\ ${ }^{1}$ Department of Materials and Production Engineering. University 'Federico II' of Naples. Piazzale Tecchio, \\ 80 - 80125 Napoli, Italy \\ 2Institute of Composite and Biomedical Materials. National Research Council. Piazzale Tecchio, 80 - 80125 Napoli, Italy \\ ${ }^{3}$ FIAT Research Center, Strada Torino, 50 - 10043 Orbassano, TO Italy
}

Received 26 May 2007; accepted in revised form 9 July 2007

\begin{abstract}
Triangular gold nano-plates have been synthesized by thermal decomposition of Au(I) dodecyl-mercaptide (i. e., $\mathrm{AuSC}_{12} \mathrm{H}_{25}$ ) dissolved in poly(vinyl acetate). Such special shape was achieved because of the ability of polymer sidegroups (i. e., the acetate groups) to be selectively absorbed on the most acid faces of the growing gold nanocrystals, thus inhibiting crystal development along these crystallographic directions. Nano-plates had an average edge length of ca. $30 \mathrm{~nm}$ and a thickness of a few nanometers.
\end{abstract}

Keywords: nanomaterials, triangular nano-plates, mercaptides, thermolysis, shape-control

\section{Introduction}

Physical properties of nano-sized metals, like surface plasmon resonance, luminescence, superparamagnetism, etc., are strictly related to the microstructure [1]. Consequently, the characteristics of nanoscopic metals can be simply tuned by modifying their morphology (i. e., the particle size and shape). Size-control has represented the first approach to achieve specific physical characteristics, but also shape-control can be used for the same purpose [2]. The dependence of physical properties on particle shape makes this second approach as the most convenient to tailor specific nanostructure properties. In the last decades, shape-control has represented a great challenge for researchers working in the nanoscience area, but it is quite difficult to obtain and it has been achieved only in few cases by solution-chemistry routes based on adequate capping agents [2].
Recently, a novel in situ chemical route for the synthesis of metal nanoparticles confined in polymeric matrices has been developed [3]. Such approach is based on the thermal decomposition of special metal precursors (namely homoleptic mercaptides) dissolved into a polymeric medium. In this paper, the isothermal decomposition of gold dodecyl-mercaptide in poly(vinyl acetate) at a temperature of $160^{\circ} \mathrm{C}$ has been investigated. This polymeric growth medium for the metal phase has shown a certain ability to influence crystal development, originating a differential growth in the crystallographic directions. In particular, the polymer sidegroups are selectively adsorbed on specific crystal faces (the most acid one) [4], thus inhibiting the development of these crystal faces, while the lessacid faces may grow by progressive deposition of gold atoms. However, such selective 'poisoningeffect' produced by the poly(vinyl acetate) sidegroups can be well evidenced only after that a significant growth of gold crystals has occurred. Con-

*Corresponding author, e-mail: giancaro@unina.it

(C) BME-PT and GTE 
sequently, the formation of simple polyhedral structures (nano-prisms) is observed in the system when gold nanocrystals have reached a size of several tens of nanometers. To the best of our knowledge, it is the fist time that a solid-state approach for the shape-controlled synthesis of triangular gold nanoplatelets has been described.

\section{Experimental}

The thermolytic organic precursor used to generate gold atoms inside the polymeric phase was $\mathrm{Au}(\mathrm{I})$ dodecyl-mercaptide (i. e., $\mathrm{AuSC}_{12} \mathrm{H}_{25}$ ) [5]. This chemical compound was synthesized by treating an ethanol solution of gold tetracholoauric acid ( $\mathrm{HAuCl}_{4}$, Aldrich) with an ethanol solution of 1-dodecane-thiol $\left(\mathrm{C}_{12} \mathrm{H}_{25} \mathrm{SH}\right.$, Aldrich) at room temperature under stirring. The obtained light-yellow solid precipitate was separated by vacuum-filtration, purified by washing with acetone, and then dissolved/dispersed in a chloroform solution of poly (vinyl acetate) $\left(M_{w}=245000 \mathrm{~g} \cdot \mathrm{mol}^{-1}\right.$, Aldrich). Thin films of dry poly(vinyl acetate)/
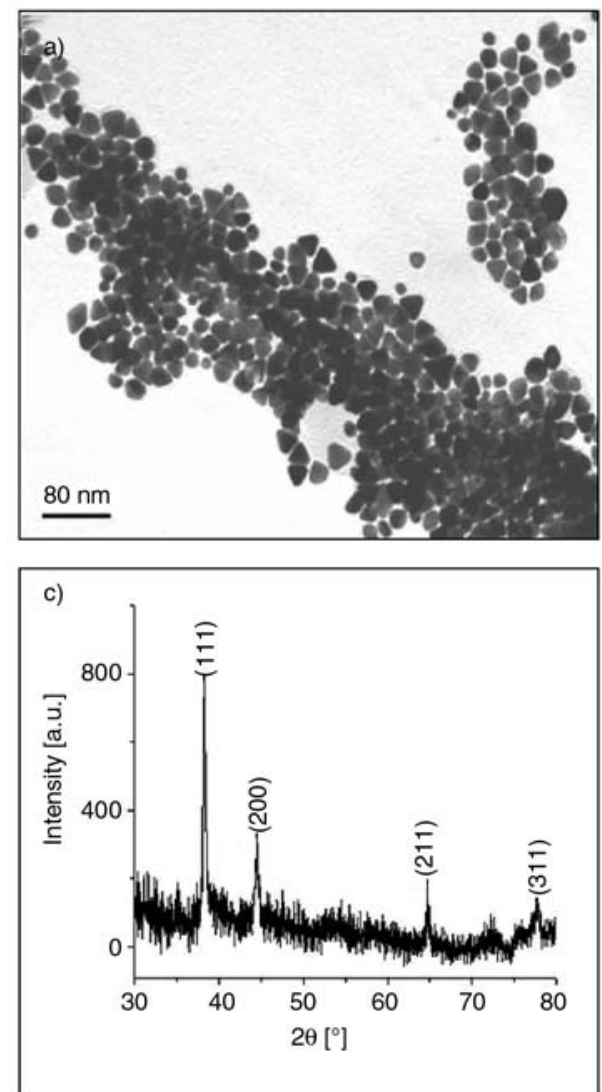

gold-mercaptide blends, containing $10 \%$ by weight of mercaptide, were obtained by solution casting from chloroform: it is worth to mention that the chloroform evaporation rate is a parameter to be set as well in order to achieve an homogeneous spatial distribution of the mercaptide inside the polymeric matrix . Gold nano-crystals were generated in the polymer phase by thermal decomposition of gold mercaptide under isothermal conditions. In particular, the heat treatment was performed at a temperature of $160^{\circ} \mathrm{C}$. To avoid temperature gradients in the thermally annealed blends, the specimens were shaped in form of films which were placed between two preheated metallic plates (at ca. $160^{\circ} \mathrm{C}$ ) to have the same temperature value on both specimen surfaces.

The obtained polymer-embedded nano-crystals were characterized by Transmission Electron Microscopy (TEM, Philips EM2085), Atomic Force Microscopy (AFM, Nanoscope IIIA, Digital Instruments), X-Ray Diffraction (XRD, Rigaku DMAX-III C), and Optical Spectroscopy (UV-VisNIR Spectrophotometer, HP 8453).
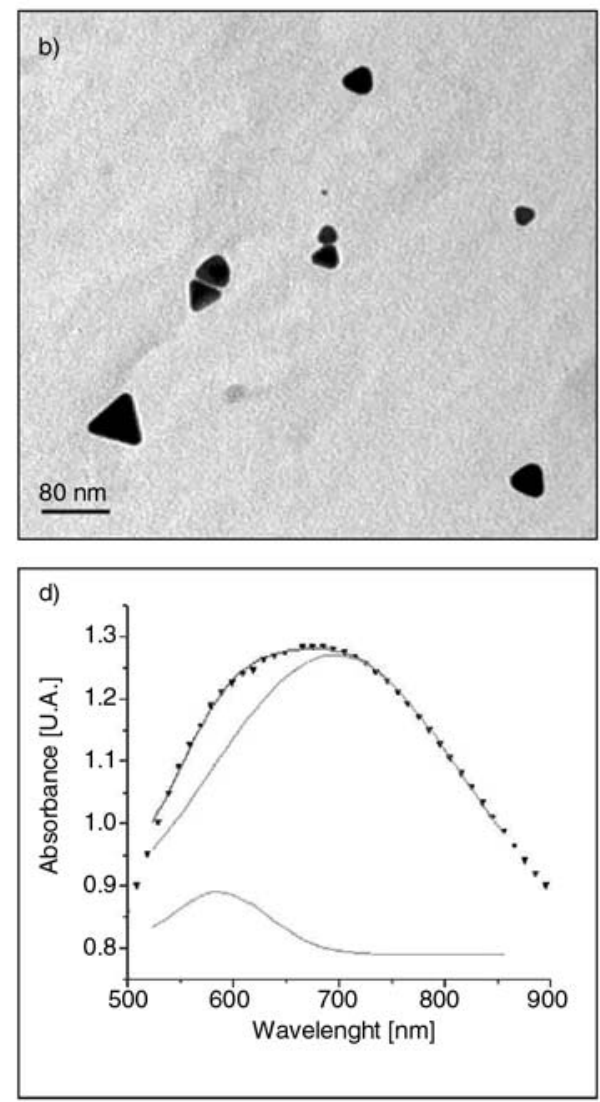

Figure 1. Gold nano-crystals characterization data: TEM-micrograph of variously shaped nano-crystals after separation/fractioning by centrifugation (a), TEM-micrograph of some isolated triangular nano-plates (b), XRD-diffraction pattern (c), and UV-Visible spectrum (d) (experimental data (४); experimental Gaussian fit (solid black line); deconvolution of experimental data (grey lines)) 


\section{Results}

Figure 1 shows the microstructure of triangular gold nano-plates which resulted after isothermal annealing of 30 minutes at $160^{\circ} \mathrm{C}$. As visible, the particles had a regular shape, consisting of simple polyhedrons, mainly made of triangular and square nano-platelets (prismatic crystals with triangular and square basal-planes). Since an uniform contrast characterized the crystal image, the presence of pyramidal geometry should be excluded. Both isolated and aggregated nanocrystals were present in the films. The average edge length of triangular nano-plates was of ca. $30 \mathrm{~nm}$ but a broad size-distribution characterized all samples. The thickness of nano-platelets cannot be estimated from TEM pictures but it was established by Atomic Force Microscopy (AFM). In particular, polymer-embedded gold nano-crystals were dissolved in chloroform and separated by centrifugation $(6,000 \mathrm{rpm})$. Nanocrystals were dispersed in chloroform and a suspension drop was placed on a mica film for AFM observation. A thickness of ca. $2 \mathrm{~nm}$ can be measured by AFM investigation of the aggregated triangular nano-crystals, as the Figure 2 shows. In addition to the large nano-crystals, a low percentage of spherical gold clusters with a size inferior to $10 \mathrm{~nm}$ was also present in the samples.

Figure 1c shows the XRD-spectrum of polymerembedded nano-plates. Owing to the amorphous nature of poly(vinyl acetate), only the diffraction pattern of gold phase was visible. The spectrum included tight peaks since the metallic phase had an average size of several nanometer tens. The most intensive peak was corresponding to the [111] plane, which is therefore the most developed crystallographic direction of triangular nano-plates [6]: such structural feature is quite common for metallic nano-plates, in fact similar structures have been found in the cases of silver, nickel, and copper nano-plates [7].

Optical properties of gold nano-crystals have been studied after their extraction from the polymeric matrix. To limit light-scattering phenomena produced by the large crystals, which significantly decreased the signal to noise ratio, optical spectra were recorded from quite dilute suspensions in chloroform. Figure 1d shows the typical optical spectrum of a gold nano-plate sample. The spectrum included a quite broad and intensive absorption band located at about $600 \mathrm{~nm}$, which is

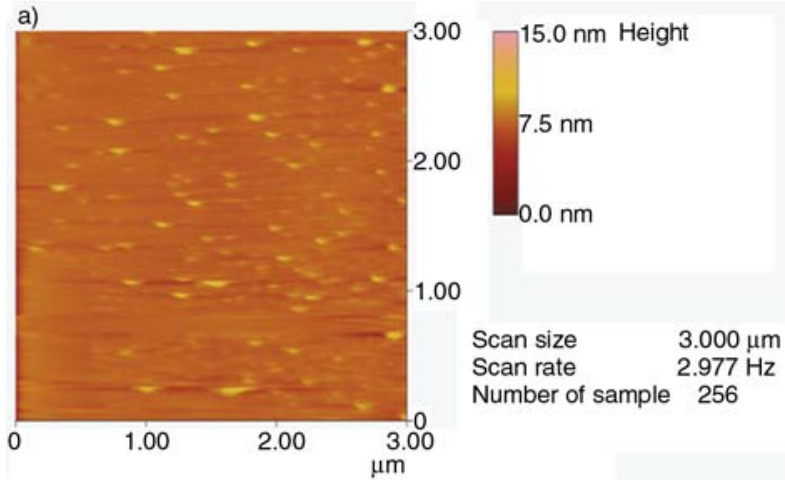

b)

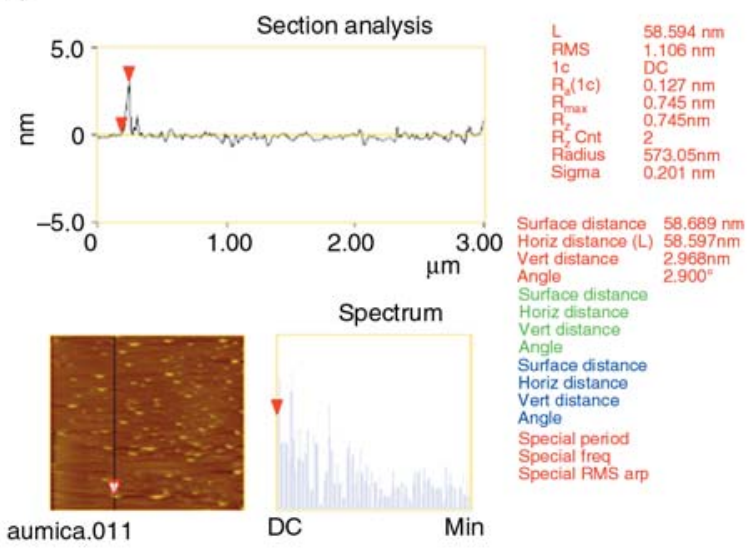

Figure 2. AFM image of gold nano-plates (a) and section analysis (b)

produced by the surface plasmon resonance of the nano-sized metallic phase. The convolution of this broad signal gives two distinct absorption bands. The band located at $580 \mathrm{~nm}$ can be attributed to the surface plasmon absorption of the low percentage of spherical nanoparticles present in the sample, whereas the absorption peak located at ca. $700 \mathrm{~nm}$ should be assigned to the in-plane quadrupole mode of gold triangular nano-plates [8].

\section{Discussion}

In general, an aspect of a fundamental importance to achieve nano-sized metal phases is to prevent aggregation in the produced solid. Particle aggregation can be avoided by generating the metal in a high viscous medium. Molten thermoplastic polymers have a very high viscosity and therefore they are ideal matrices for the nucleation/growth of nanometric metals. The polymeric medium should have a glass transition temperature $\left(T_{g}\right)$ lower than the decomposition temperature of the metal precursor. In addition to the high viscosity also a certain ability to be absorbed on the metal surface is required for the polymer, in order to avoid metal 
segregation and therefore its aggregation. When the metal phase is generated by thermal decomposition of homoleptic mercaptides, amorphous polystyrene represents a quite good material for cluster formation/growth since the glass transition temperature is not too far below the mercaptide decomposition temperature and the ability for the polymer sidegroups to be lightly absorbed on the electrophilic metal surface (probably by $\pi$-electron density donation from phenyl groups to the metal). However, the faces of a metal crystal have a different ability to bond nucleophilic species, because the acidity of absorption sites depends on the metal coordination number. The quite low ability of polystyrene side-groups to be absorbed on the crystal faces is not enough to discriminate between them. Owing to the ester functions in the poly(vinyl acetate) side-groups, these molecules are preferentially absorbed on the most acid faces of the metallic crystals. Polymer absorption creates a diffusion barrier on these crystal faces, thus inhibiting their development and simplifying the polyhedral geometry. To observe such a differential growth of the crystal faces a significant crystal development is required. For such a reason, mercaptide should be slowly decomposed to generate the metal atoms required to grow nuclei by surface deposition. In the growth of triangular gold plates, thermal decomposition was performed at $160^{\circ} \mathrm{C}$ and the annealing treatment required more than 30 minutes to allow significant growth of metal crystals with a differential development of crystal faces. Finally, according to the LaMer model for monodispersed particle formation, a single nucleation stage must take place during the process, and then the generated nuclei should grow by addition of gold atoms to the crystal surface. During the growth stage the most acid faces do not significantly develop because of polymer absorption, leaving to a simple geometrical figure.

\section{Conclusions}

The possibility to obtain triangular gold nanoplates by an in situ synthesis based on mercaptide thermal decomposition in polymer medium has been investigated. The selection of polymer with adequately capping side-groups allows to simplify the shape of polyhedral crystals by inhibiting the development of most acid crystallographic faces. As a result, the poly(vinyl acetate) with has shown to be a good growth medium to achieve triangular nano-plates.

\section{References}

[1] Burda C., Chen X.: Chemistry and properties of nanocrystals of different shapes. Chemical Review, 105, 1025-1102 (2005).

[2] Sun Y. G., Xia Y. N.: Shape-controlled synthesis of gold and silver nanoparticles. Science, 298, 2176 2179 (2002)

[3] Carotenuto G., Martorana B., Perlo P., Nicolais L.: A universal method for the synthesis of metal and metal sulfide clusters embedded in polymer matrices. Journal of Material Chemistry, 13, 2927-2930 (2003).

[4] Tannenbaum R., Reich S., Flenniken C. L., Goldberg E. P.: Shape control of iron oxide nanoclusters in polymeric media. Advanced Materials, 19, 1402-1405 (2002).

[5] Carotenuto G., Longo A., Nicolais L.: Synthesis of molecular gold-clusters with luminescence properties by mercaptide thermolysis in polymer matrices. International Journal of Nanoscience, 6, 65-69, (2007).

[6] Wiley B., Sun Y., Mayers B., Xia Y.: Shape-controlled synthesis of metal nanostructures: the case of silver. Chemistry-A European Journal, 11, 454-463 (2005).

[7] Chen S., Carroll D. L.: Synthesis and characterization of truncated triangular silver nanoplates. Nano Letters, 2, 1003-1007 (2002).

[8] Malikova N., Pastoriza-Santos I., Schierhorn M., Kotov N. A.: Layer-by-layer assembled mixed spherical and planar gold nanoparticles: control of interparticle interactions. Langmuir, 18, 3694-3697 (2002). 